\title{
TRIM67 promotes NF-кB pathway and cell apoptosis in GA-13315-treated lung cancer cells
}

\author{
RUI LIU ${ }^{1,2}$, YAJUAN CHEN ${ }^{1}$, TAO SHOU ${ }^{2}$, JING HU $^{2}$, JINGBO CHEN $^{3}$ and CHEN QING ${ }^{1}$ \\ ${ }^{1}$ School of Pharmaceutical Science \& Yunnan Key Laboratory of Pharmacology for Natural Products, Kunming Medical \\ University, Kunming, Yunnan 650031; ${ }^{2}$ Department of Oncology, The First People's Hospital of Yunnan Province, Kunming, \\ Yunnan 650032; ${ }^{3}$ Key Laboratory of Medicinal Chemistry for Natural Resource, Ministry of Education, \\ School of Chemical Science and Technology, Yunnan University, Kunming, Yunnan 650031, P.R. China
}

Received November 7, 2018; Accepted June 25, 2019

DOI: $10.3892 / \mathrm{mmr} .2019 .10509$

\begin{abstract}
Chlorine-3,15-dioxy-gibberellic acid methyl ester (GA-13315), a gibberellin derivative, possesses strong anti-tumor activity in vitro and in vivo. The present study aimed to investigate the underlying mechanisms of GA-13315-induced apoptosis in human non-small cell lung cancer cell lines. Lung cancer cells were treated with different doses of GA-13315 (4, 8, 16 and $32 \mathrm{ng} / \mu \mathrm{l})$ for $48 \mathrm{~h}$, and a CCK8 assay was performed to measure cell viability. Alteration in gene expression was identified using RNA-sequencing (RNA-Seq). Quantitative polymerase chain reaction (qPCR) was used to confirm the differentially expressed genes (DEGs) identified in RNA-Seq. Gene expression plasmids or small interfering RNA were used to overexpress or silence targeted genes, in order to investigate downstream signals. Chromatin immunoprecipitation was conducted to evaluate the binding of transcription factors to the target genes. A Student's t-test or one-way analysis of variance followed by Tukey's honestly significant difference post-hoc test were performed to evaluate the significance between groups. $\mathrm{P}<0.05$ was considered to indicate a statistically significant difference. GA-13315 significantly decreased the number of viable cells
\end{abstract}

Correspondence to: Dr Chen Qing, School of Pharmaceutical Science \& Yunnan Key Laboratory of Pharmacology for Natural Products, Kunming Medical University, 1168 Chunrong West Road, Kunming, Yunnan 650031, P.R. China

E-mail: 422686650@qq.com

Dr Jingbo Chen, Key Laboratory of Medicinal Chemistry for Natural Resource, Ministry of Education, School of Chemical Science and Technology, Yunnan University, 2 Cuihu North Road, Kunming, Yunnan 650031, P.R. China

E-mail: chen196809@outlook.com

Abbreviations: NSCLC, non-small-cell lung cancer; EGF, epithelial growth factor; FC, fold change; PVDF, polyvinylidene difluoride

Key words: GA13315, tripartite motif containing 67, NF-кB p100 subunit, Fas cell surface death receptor, apoptosis and induced apoptosis among lung cancer cells (median lethal dose $=12-16 \mathrm{ng} / \mu \mathrm{l}$ ). RNA-Seq identified 250 significant DEGs, including 94 upregulated and 156 downregulated genes in A549 cells $(\mathrm{P}<0.05$; fold change $\geq 1.5)$. Upregulation of TRIM67, NF- $\kappa$ B subunit $2(\mathrm{NF}-\kappa \mathrm{B} 2)$ and FAS was additionally confirmed using qPCR and western blot analysis in A549 and H460 cells. Apoptosis of A549 cells was significantly decreased following knockdown of TRIM67. GA-13315 promoted TRIM67 expression to increase FAS expression and cell apoptosis. TRIM67 promoted the processing of NF- $\kappa \mathrm{B} 2$ into its active form, p52, which then enhanced the $N F-\kappa B$ pathway and GA-13315-induced apoptosis.

\section{Introduction}

Lung cancer is the primary cause of cancer-associated mortalities worldwide, and non-small-cell lung cancer (NSCLC) constitutes $85 \%$ of all cases of lung cancer $(1,2)$. Platinum combined with paclitaxel, gemcitabine and pemetrexed is currently the basic chemotherapy regimen for lung cancer, but it has a low efficacy and strong side effects (3). Patients with epidermal growth factor (EGF) receptor, anaplastic lymphoma kinase or other cancer-driving gene mutations initially benefit from targeted therapy, but subsequently experience severe relapse and drug resistance (4). Therefore, there is an urgent need for the development of new drugs for patients with lung cancer.

Gibberellin is a triterpene compound. It was originally isolated from the induced Bakanae gibberella to yield an amorphous solid. It is a broad-spectrum plant-growth regulator, which promotes the growth and development of crops. Gibberellin is widely distributed in the metabolites of fungi and higher plants. It is also widely used in vegetable cultivation, agriculture and horticulture (5).

13-Chlorine-3,15-dioxy-gibberellic acid methyl ester (GA-13315), a small molecular compound (molecular weight 370.4) derived from gibberellin, possesses strong anti-angiogenic and anti-cancer effects. It inhibited recombinant human EGF-induced chemotactic motility and capillary-like tube formation in primary cultured human endothelial cells (6). GA-13315, at sub-toxic concentrations, reversed the multi-drug resistance mediated by ATP binding 
cassette subfamily B member 1 (7). It inhibited proliferation and caused apoptosis in oral cancer (8). The product of the protein phosphatase 2 regulatory subunit Bbeta (PPP2R2B) gene belongs to the phosphatase II regulation subunit B family, which serves important roles in cell growth and division (9). PPP2R2B methylation is associated with survival and prognosis in patients with gliomas (10). GA-13315 also suppressed PPP2R2B expression in H1299 lung cancer cells (8). In vivo studies have indicated that it had a significant inhibitory effect on the growth of human colon cancer xenografts in HCT116 nude mice without causing any apparent side effects (6).

Therefore, GA-13315 exhibited marked potential as a novel therapeutic agent for lung cancer. However, the underlying mechanism of GA-13315-mediated apoptosis remains unclear. The present study used RNA-sequencing (RNA-Seq) to survey the differentially expressed genes (DEGs) between cancer cells treated with control vehicles and those treated with GA-13315. The results indicated that GA-13315 increased tripartite motif containing 67 (TRIM67), NF- $\kappa \mathrm{B}$ subunit 2 $(\mathrm{NF}-\kappa \mathrm{B} 2)$ and Fas cell surface death receptor (FAS) expression. For example, TRIM67 has been demonstrated to be downregulated in NSCLC cancer cell lines (11). Genetic variations of NF- $\kappa \mathrm{B} 2$ are significantly associated with NSCLC risk and overall survival (12). FAS regulates lung cancer cell apoptosis $(13,14)$. Additional investigation indicated that TRIM67 promoted the processing of $\mathrm{NF}-\kappa \mathrm{B} 2$ into its active form, $\mathrm{p} 52$, to enhance the NF- $\kappa \mathrm{B}$ pathway, which serves an important role in GA-13315-induced apoptosis.

\section{Materials and methods}

Cell culture. A549 and H460 lung carcinoma cell lines were obtained from the Kunming Institute of Zoology, Chinese Academy of Sciences, and cultured in high glucose Dulbecco's modified Eagle's medium (DMEM; GE Healthcare) containing $10 \%$ fetal bovine serum (FBS, EMD Millipore) and $1 \%$ penicillin-streptomycin (EMD Millipore) in a $37^{\circ} \mathrm{C}$ incubator with $5 \% \mathrm{CO}_{2}$ (Thermo Fisher Scientific, Inc.). Cells were grown to $70 \%$ confluence prior to use for subsequent experiments.

Cell viability assay. GA-13315 (purity >95\%, measured by proton nuclear magnetic resonance spectroscopy) was prepared by the School of Chemical Science and Technology at Yunnan University (Kunming, China). Cell proliferation, with or without GA-13315 treatment, was measured using a Cell Counting Kit-8 (CCK-8) assay kit (BD Biosciences). Briefly, cells were seeded into 96-well plates at 2,000 cells/plate, incubated overnight and treated with various doses $(4,8,16$ and $32 \mathrm{ng} / \mu \mathrm{l}$ ) of GA-13315 or the vehicle control for $48 \mathrm{~h}$. Then, $10 \mu$ l CCK8 solution was added to each well and cells were incubated for an additional $2.5 \mathrm{~h}$. Optical density was measured by a microplate reader (Thermo Fisher Scientific, Inc.) at a wavelength of $450 \mathrm{~nm}$.

$R N A$-Seq. In total, $2 \mathrm{ml}$ A549 cells $\left(2 \times 10^{5}\right.$ cells $\left./ \mathrm{ml}\right)$ were plated into a 6-well plate in triplicate. A total of 3 wells were treated with $16 \mathrm{ng} / \mu \mathrm{l} \mathrm{GA}-13315$ (treated), and the other 3 wells treated with control vehicle (dimethyl sulfoxide; DMSO) at $37^{\circ} \mathrm{C}$ for $48 \mathrm{~h}$. RNA was extracted with a RNeasy kit (Invitrogen; Thermo Fisher Scientific, Inc.) according to the manufacturer's protocol. RNA libraries were prepared using an Illumina NEB Next Ultra RNA Library Prep Kit (New England Biolabs, Inc.; cat. no. E7530). Briefly, replicates of $5 \mu \mathrm{g}$ total RNA were sheared into fragments (200 nt) and reverse transcribed into cDNA, which were then blunt-ended and phosphorylated followed by the addition of single 3 ' adenosine moiety and ligated with Illumina adapters. Libraries were amplified by polymerase chain reaction (PCR) using a NEB Phusion Polymerase (New England Biolabs, Inc.) followed by purification. The thermocycling conditions were as follows: Initial denaturation at $98^{\circ} \mathrm{C}$ for $30 \mathrm{sec}$; followed by 13 cycles of $98^{\circ} \mathrm{C}$ for $10 \mathrm{sec}, 65^{\circ} \mathrm{C}$ for $30 \mathrm{sec}, 72^{\circ} \mathrm{C}$ for $30 \mathrm{sec}$ and by a final extension step at $65^{\circ} \mathrm{C}$ for $5 \mathrm{~min}$. The primers used to amplify the libraries were the following: Forward primer, 5'-AATGAT ACGGCGACCACCGA-3'; and reverse primer, 5'-CAAGCA GAAGACGGCATACGA-3'. RNA-Seq was conducted using paired-end, 100 base pair reads by HiSeq 2500 v4 100PE (Illumina, Inc.).

DEGs. Subsequent to filtering out the low quality reads, the remaining reads were aligned to the reference genome hg19 using TopHat2 software (v2.0.8: http://tophat.cbcb.umd.edu/) with default parameters (15). Fragments per kilobase of exon model per million fragments mapped was used to quantify the expression of each gene. Differentially expressed genes were identified using Cuffdiff software (v2.1.0: http://coletrapnell-lab.github.io/cufflinks/) by comparing the GA-13315 treated group to the control vehicle treated group. Genes that passed the $\mid \log 2$ fold change $(\mathrm{FC}) \mid>1.5$ and $\mathrm{P}<0.05$ thresholds were considered to be significant DEGs (16). Pathway analysis was performed using Ingenuity Pathway Analysis tools (version 2018; Qiagen Bioinformatics).

Quantitative (q)PCR. A549 or H460 cells were plated in triplicate into a 6-well plate. A total of 3 wells of cells were treated with $16 \mathrm{ng} / \mu \mathrm{l} \mathrm{GA}-13315$ (Treated) and the other three wells of cells treated with control vehicle (DMSO) at $37^{\circ} \mathrm{C}$ for 48 h. RNA was extracted with RNeasy kit (Invitrogen; Thermo Fisher Scientific, Inc.). Equal amounts of cDNA were synthesized using the Advantage RT-for-PCR kit (Clontech, Mountain View, CA). Briefly, the samples were mixed with random primers and incubated at $65^{\circ} \mathrm{C}$ for $5 \mathrm{~min}$ and then on ice for at least $1 \mathrm{~min}$. Reverse transcriptase was added to each tube, mix and incubate at $25^{\circ} \mathrm{C}$ for $10 \mathrm{~min}$ and then $42^{\circ} \mathrm{C}$ for $50 \mathrm{~min}$, heat inactivated at $70^{\circ} \mathrm{C}$ for $15 \mathrm{~min}$, and then kept on ice. A total of $1 \mathrm{ul} \mathrm{RNase} \mathrm{H}$ was added and the samples were incubated at $37^{\circ} \mathrm{C}$ for $20 \mathrm{~min}$. The 1 st strand cDNA was stored at $-20^{\circ} \mathrm{C}$ until use for $\mathrm{qPCR}$.

qPCR was performed using Power SYBR-Green PCR Master Mix (Applied Biosystems; Thermo Fisher Scientific, Inc.). All qPCR performed using SYBR Green was conducted at $50^{\circ} \mathrm{C}$ for $2 \mathrm{~min}$ and $95^{\circ} \mathrm{C}$ for $10 \mathrm{~min}$, and then 40 cycles of $95^{\circ} \mathrm{C}$ for $15 \mathrm{sec}$ and $60^{\circ} \mathrm{C}$ for $1 \mathrm{~min}$. The specificity of the reaction was verified by melt curve analysis. GAPDH was used an internal control and all data were normalized to GAPDH. Control groups for the TRIM67 siRNA transfection and TRIM67 plasmid assays used negative control siRNA and control empty vectors, respectively. The relative gene expression was calculated using $2^{-\Delta \Delta \mathrm{Cq}}$ quantification method (17). The primers used are summarized in Table SI. 
Transient transfection. A set of siRNAs targeting TRIM67 (5'-GTACCATCGACGGTCTTCA-3'; 5'-CCTCGTTGCTCA GTGTGAT-3'; 5'-GCGGAGTTTGATCTGACTT-3'; Shanghai GenePharma Co., Ltd.) targeting TRIM67 or fluorescein amidite-labeled control siRNA (5'-TAAGGCTATGAAGAG ATAC-3'; cat. no., A07001; Shanghai GenePharma Co., Ltd.) was dissolved in $\mathrm{ddH}_{2} \mathrm{O}$ to a final concentration of $0.05 \mathrm{nmol} / \mathrm{l}$, aliquoted and stored at $-20^{\circ} \mathrm{C}$. Cells in the logarithmic growth phase were plated into 6 -well plates at $1 \times 10^{5}$ cells per well. When the cells reached $60-70 \%$ density, transfection with $10 \mathrm{nM}$ siRNA was performed using Lipofectamine (Invitrogen; Thermo Fisher Scientific, Inc.) according to the protocol of the manufacturer. The transfection efficiency of control siRNA was visually inspected under a fluorescence microscope (magnification, x200). Culture medium (90\% DMEM + 10\% FBS) was replaced $6 \mathrm{~h}$ after transfection. A total of $48 \mathrm{~h}$ after transfection, cells were harvested and RNA or protein was extracted for analysis.

For the gene overexpression assays, whole-length TRIM67 was synthesized and inserted into pcDNA3.1 expression vector (GenScript). An empty vector was used as a control. When the cells were at $60-70 \%$ confluence, gene transfection (1 $\mu \mathrm{g}$ plasmid) was performed using Lipofectamine, as aforementioned. Transfection efficiency was evaluated through visual inspection under a fluorescence microscope (magnification, $\mathrm{x} 200$ ) by transfecting $1 \mu \mathrm{g}$ expression vector (pEGFP-N1; Clontech Laboratories, Inc.) containing green fluorescent protein. A total of $48 \mathrm{~h}$ after transfection, cells were harvested and RNA or protein was extracted for analysis. The transfection efficiency of knockdown or overexpression was additionally quantified by measuring the density of the band of western blot analysis using ImageJ software (v1.8.0; National Institutes of Health) (Fig. S1).

Cell apoptosis assay. Cells were transfected and GA-13315 $(16 \mathrm{ng} / \mu \mathrm{l})$ was added $24 \mathrm{~h}$ after transfection. At $48 \mathrm{~h}$ after transfection, cells were harvested for the apoptosis assay with FITC Annexin V Apoptosis Detection Kit I (BD Biosciences). Briefly, adherent cells were trypsinized, transferred to an Eppendorf tube and collected via centrifugation for $10 \mathrm{~min}$ at $4^{\circ} \mathrm{C}$ at $300 \mathrm{x} \mathrm{g}$. Cell pellets were washed twice with pre-cooled PBS and rinsed once with $1 \mathrm{X}$ binding buffer (10X; double vapor dilution). Cells were additionally suspended in PBS to a concentration of $1 \times 10^{6} / \mathrm{ml}$, following which $100 \mu \mathrm{l}$ cell suspensions $\left(1 \times 10^{5}\right)$ were transferred to centrifuge tubes, mixed with $5 \mu \mathrm{l}$ fluorescein isothiocyanate-Annexin $\mathrm{V}$ and $10 \mu \mathrm{l}$ propidium iodide, and incubated for $15 \mathrm{~min}$ at room temperature in the dark. Then, $400 \mu \mathrm{l} 1 \mathrm{X}$ binding buffer was added to the cells $1 \mathrm{~h}$ prior to flow cytometry. The data were analyzed using FlowJo software (version 10; BD Biosciences). To investigate the role of NF- $\kappa$ B pathway in GA-13315 induced apoptosis, A549 cells $\left(2 \times 10^{5}\right.$ cells $\left./ \mathrm{ml} ; 2 \mathrm{ml}\right)$ were plated into a 6 -well plate in triplicate and incubated in a cell culture incubator at $37^{\circ} \mathrm{C}$ and $5 \% \mathrm{CO}_{2}$. After $24 \mathrm{~h}$, cells were treated with control vehicle, GA-13315 (16 ng/ $\mu \mathrm{l}$ ), and GA-13315 plus $500 \mu \mathrm{M} \mathrm{NF- \kappa B}$ essential modulator-binding domain (NBD; ABclonal, Inc.) for $48 \mathrm{~h}$. Cells were subsequently harvested to measure the level of apoptosis.

Western blot analysis. Total protein from the A549 cells was extracted using radioimmunoprecipitation assay lysis buffer (Beyotime Institute of Biotechnology) and quantified using a BCA
Protein Assay kit (Beyotime Institute of Biotechnology). Equal amounts of proteins $(15 \mu \mathrm{g})$ were separated via $10 \%$ SDS-PAGE and transferred onto a $0.45 \mu \mathrm{m}$ polyvinylidene difluoride (PVDF) membrane (EMD Millipore) through a wet trans-blot system (Bio-Rad Laboratories, Inc.). The membranes were then blocked for $1 \mathrm{~h}$ at room temperature in 5\% nonfat dried milk diluted in TBST (Bio-Rad Laboratories, Inc.), incubated overnight at $4^{\circ} \mathrm{C}$ with rabbit polyclonal antibodies against TRIM67 (1:250; cat. no., PA5-42274; Thermo Fisher Scientific, Inc.), Fas (1:1,000; cat. no., ab82419; Abcam), or NF-кB2 (1:2,000; cat. no., 37359; Cell Signaling Technology), and then with a goat anti-rabbit horseradish peroxidase-conjugated secondary antibody $(1: 3,000$; cat. no., 12-348; Sigma-Aldrich; Merck KGaA) for $1 \mathrm{~h}$ at room temperature, and analyzed using an enhanced chemiluminescence (ECL) detection kit (Applygen Technologies, Inc.). $\beta$-actin (1:2,000; cat. no. ab8227; Abcam) was used as the internal control. The density of the bands was analyzed using ImageJ software (v1.8.0; National Institutes of Health).

Dual luciferase report assay. Wild type FAS promoter (-1,025 to $+25 \mathrm{bp}$ ) or mutant (without NF-kB binding sites) was synthesized and inserted into a pGL3-Basic vector (GeneScript). A FAS promoter activity assay was performed using a dual luciferase reporter system (Promega Corporation), according to the manufacturer's protocol. Briefly, 10,000 cells in $0.5 \mathrm{ml}$ DMEM culture medium (GE Healthcare) were seeded into 24-well plates and incubated at $37^{\circ} \mathrm{C}$ until the cells had attached. Each well was co-transfected with $200 \mathrm{ng}$ luciferase plasmids (pGL3-Basic plasmids without the promotor region as the negative control or plasmids containing FAS promoter with/without NF- $\mathrm{KB}$ binding sites) and $5 \mathrm{ng}$ pRL-TK plasmids (Promega Corporation) encoding Renilla, which was used as the internal control to assess transfection efficiency. At $24 \mathrm{~h}$ after transfection, whole-cell lysates were collected and measured using a GloMax Microplate Luminometer (Promega Corporation). Transfections were performed in triplicate and then repeated 3 times to assure reproducibility. All luciferase reads were normalized to the corresponding Renilla value. Relative luciferase activity was calculated using the following formula: Relative luciferase levels=Luc-promoter/Luc-pGL3 basic.

Chromatin immunoprecipitation (ChIP) assay. A ChIP assay was performed with a ChIP-IT kit (cat.no. 53040; Active Motif). A total of $1 \times 10^{7}$ cells were cross-linked and lysed. Chromatin was extracted and sonicated to $200-800$ bp length fragments with 8 rounds of $10 \mathrm{sec}$ pulses using $25 \%$ power. Normalized inputs of sheared chromatin DNA were incubated with $3 \mu \mathrm{g}$ negative control immunoglobulin G (1:30; cat. no. ab171870; Abcam) or a p52 antibody (1:30; cat. no. ab7972; Abcam) overnight at $4^{\circ} \mathrm{C}$. The immunocomplex was treated with RNase A to remove RNA and incubated at $65^{\circ} \mathrm{C}$ for $4 \mathrm{~h}$ to remove cross-links. Proteins were removed by treating with proteinase $\mathrm{K}$, and DNA was purified and subjected to PCR detection following the aforementioned protocol. Primers targeting the FAS promoter (forward, CTCCATTCTCCT TCAAGACCT; reverse, GTGTGTCACTCTTGCGCGAGAT) were used to evaluate the binding of the p52 antibody.

Statistical analysis. Data were analyzed by SPSS 19.0 software (SPSS Inc.). and are presented as the mean \pm standard 

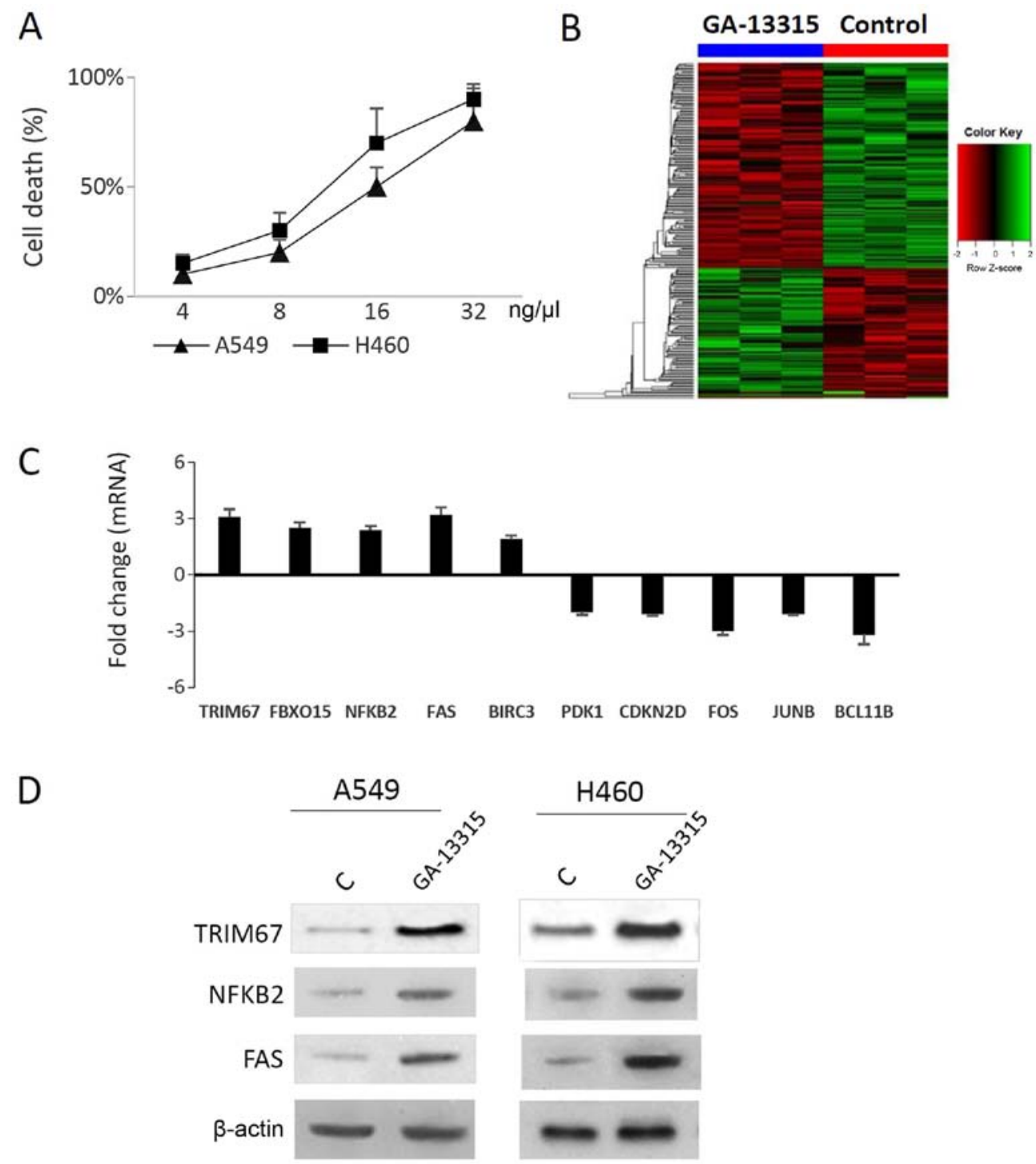

Figure 1. RNA-seq identifies several differentially expressed genes. (A) The lung cancer A549 and H460 cell lines were used to evaluate the effect of GA-13315. Cells were treated with control vehicle (DMSO) or GA-13315 for $48 \mathrm{~h}$, and cell viability was measured. Cell death rate was plotted against the GA-13315 concentration to reveal the median lethal dose for each cell line. (B) RNA-seq identified 250 differentially expressed genes $(\mathrm{P}<0.05$; fold change $>1.5$ ). Hierarchical clustering was performed and the differentially expressed genes were summarized as a heatmap. (C) Quantitative polymerase chain reaction confirmed that TRIM67, FBXO15, NF-кB2, FAS and BIRC3 were overexpressed; while PDK1, CDKN2D, FOS, JUNB and BCL11B were downregulated in GA-13315-treated A549 cells. (D) Western blot analysis. Total proteins from the vehicle control (DMSO) and GA-13315-treated cell lines were examined using

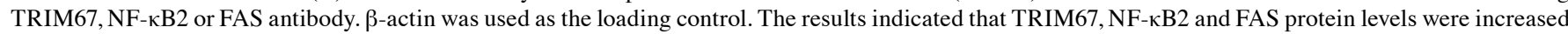
in both A549 and H460 cells. RNA-seq, RNA-sequencing; GA-13315, 13-chlorine-3,15-dioxy-gibberellic acid methyl ester; DMSO, dimethyl sulfoxide; TRIM67, tripartite motif containing 67; FBXO15, F-box protein 15; NF-кB2, NF-kB subunit 2; FAS, Fas cell surface death receptor; BIRC3; baculoviral IAP repeat containing 3; PDK1, pyruvate dehydrogenase kinase 1; CDKN2D, cyclin dependent kinase inhibitor 2D; FOS, Fos proto-oncogene, AP-1 transcription factor subunit; JUNB, JunB proto-oncogene, AP-1 transcription factor subunit; BCL11B, BAF chromatin remodeling complex subunit BCL11B.

deviation. Student's t-tests were performed to evaluate the significance of difference between two groups. Differences between multiple groups were analyzed using analysis of variance followed by Tukey's honestly significant difference post-hoc test was used to evaluate the differences between groups. $\mathrm{P}<0.05$ was considered to indicate a statistically significant difference.

\section{Results}

RNA-Seq identified TRIM67, NF- $\kappa B 2$ and FAS as differentially expressed genes induced by GA-13315. A cell viability assay indicated that GA-13315 caused $\sim 50 \%$ A549 cell death at a concentration of $16 \mathrm{ng} / \mu 1$ (Fig. 1A). Therefore, this concentration was selected for subsequent experiments. Upon GA-13315 treatment, a total of 250 genes were identified to be significantly differentially expressed $(\mathrm{FC} \geq 1.5 ; \mathrm{P}<0.05)$, of which 94 genes were upregulated and 156 genes were downregulated (Fig. 1B). A detailed list is presented in Table SII. The TRIM67, NFKB2 and FAS genes were selected for subsequent investigation as they were enriched in pathway analysis. In total, seven genes were randomly selected for qPCR verification. The expression levels of these 10 genes were additionally confirmed using qPCR, including TRIM67 (3.1), F-box protein 15 (2.5), NF-кB2 (2.4), Fas cell surface death receptor (3.2), baculoviral IAP repeat containing 3 (1.9), 

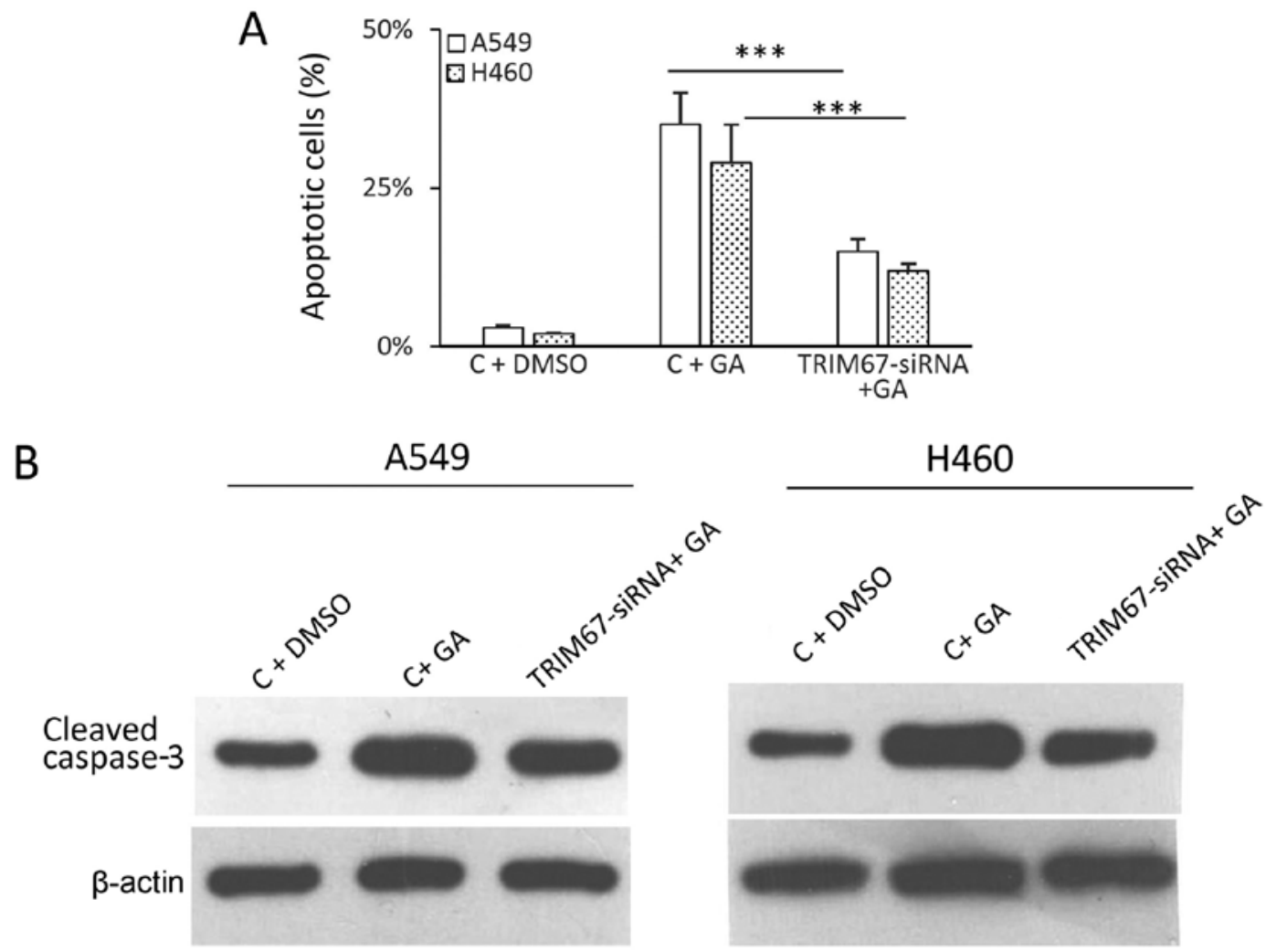

Figure 2. TRIM67 regulates GA-13315-induced apoptosis. (A) Cells were transfected with non-targeting control siRNA or TRIM67 siRNA, and treated with vehicle control or GA. The apoptosis rate was calculated by dividing Annexin V-positive cells by total cells. TRIM67 siRNA decreased GA-13315-induced apoptosis in A549 and H460 cell lines, as measured by an Annexin V assay. One way analysis of variance followed by Tukey's honestly significant difference post-hoc test was used to evaluate the difference between groups each cell line. Results are expressed as the mean \pm standard deviation. ${ }^{* * *} \mathrm{P}<0.001$. (B) TRIM67 siRNA suppressed cleaved caspase-3 levels in GA-13315-treated cells. TRIM67, tripartite motif containing 67; siRNA, small interfering RNA; GA/GA-13315, 13-chlorine-3,15-dioxy-gibberellic acid methyl ester.

pyruvate dehydrogenase kinase $1(-2)$, cyclin dependent kinase inhibitor 2D (-2.1), Fos proto-oncogene, AP-1 transcription factor subunit (-3), JunB proto-oncogene, AP-1 transcription factor subunit $(-2.1)$ and BAF chromatin remodeling complex subunit BCL11B (-3.2) (Fig. 1C). TRIM67 has been demonstrated to be downregulated in NSCLC cancer cell lines (11), and pathway analysis from the present study indicated that $\mathrm{NF}-\kappa \mathrm{B}$ and FAS pathways were involved in this process (Fig. S2). Therefore, the protein levels of TRIM67, FAS and $\mathrm{NF}-\kappa \mathrm{B} 2$ were also examined, and it was identified that all 3 were increased (Fig. 1D), thereby confirming the qPCR results.

TRIM67 mediates GA-13315-induced cell apoptosis. To investigate the association between TRIM67 and GA-13315-induced apoptosis, TRIM67 expression was silenced using siRNA and the levels of apoptosis were assessed using Annexin V staining and cleaved caspase-3. Annexin V staining indicated that silencing TRIM67 decreased the GA-13315-induced apoptosis rate from 50 to $15 \%$ in $\mathrm{A} 549$ cells $(\mathrm{P}<0.001)$ and from 35 to $12 \%$ in $\mathrm{H} 460$ cells $(\mathrm{P}<0.001$; Fig. $2 \mathrm{~A})$. Exemplary images of flow cytometry are presented in Fig. S3. This observation was additionally confirmed by western blot analysis, which indicated that the silencing of TRIM67 decreased the levels of GA-13315-induced cleaved caspase-3 in both cell lines examined (Fig. 2B). Therefore, these results suggested a role for TRIM67 in mediating GA-13315-induced apoptosis.
TRIM67 promotes $N F-\kappa B 2$ processing and enhances the $N F-\kappa B$ pathway. Pathway analysis indicated that the $N F-\kappa B$ complex was enhanced in GA-13315-treated cells. As NF- $\kappa$ B2 is a precursor protein that requires additional processing to convert into its active forms (18), and TRIM67 belongs to the TRIM family and possesses ubiquitin E3 ligase activity (19), whether TRIM67 affected NF- $\kappa$ B2 processing was examined. Forced overexpression of TRIM67 increased p52 but decreased the levels of NF- $\mathrm{B} 2$ precursor p100 (Fig. 3A), while knockdown of TRIM67 increased p100 levels but decreased p52 levels (Fig. 3B), suggesting that TRIM67 promoted NF-кB2 processing. The results from the $\mathrm{qPCR}$ analysis indicated that the alteration of TRIM67 had no effect on the NF- $\kappa$ B2 mRNA level (Fig. 3C and D), suggesting that TRIM67 regulates $\mathrm{NF}-\kappa \mathrm{B} 2$ at the protein level.

GA-13315 induces FAS upregulation and cell apoptosis via the $N F-\kappa B$ pathway. Whether the NF- $\kappa \mathrm{B}$ pathway is involved in GA-13315-induced FAS upregulation and apoptosis was then investigated. An $\mathrm{NF}-\kappa \mathrm{B}$ essential NBD peptide was used to inhibit the $N F-\kappa B$ pathway, and it was identified that it suppressed the GA-13315-induced cell apoptosis rate, decreasing it from 35 to $12 \%$ in A549 cells $(\mathrm{P}<0.001)$ and from 32 to $11 \%$ in H460 cells (Fig. 4A). The anti-apoptotic effects of NBD peptide on GA-13315-induced cell apoptosis were additionally confirmed by western blot analysis on cleaved caspase-3 (Fig. 4B). Both RNA and protein levels of FAS 
A

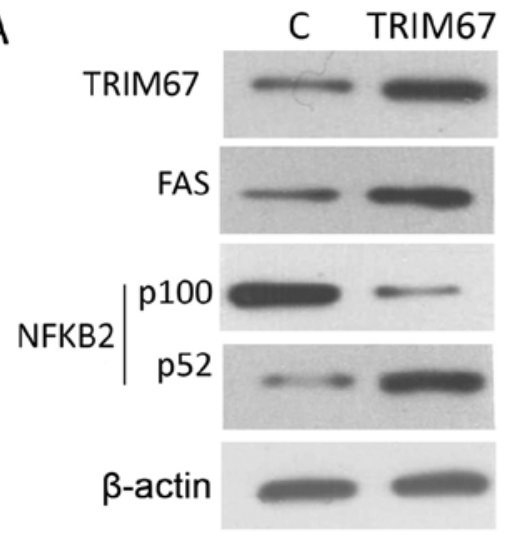

C

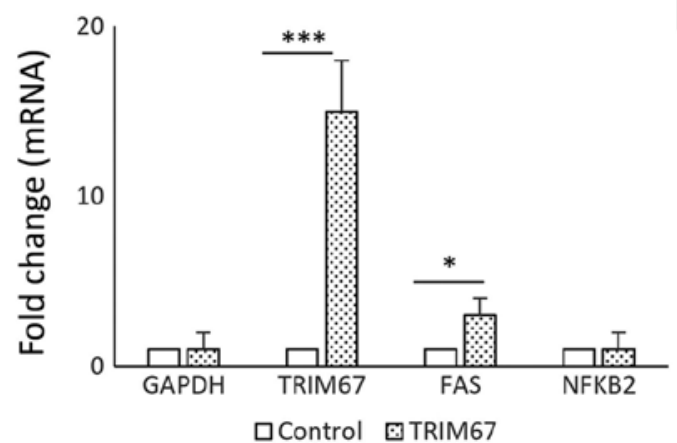

B

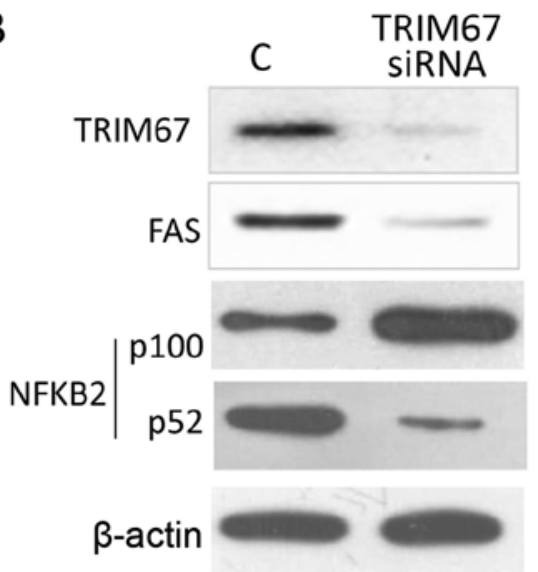

D

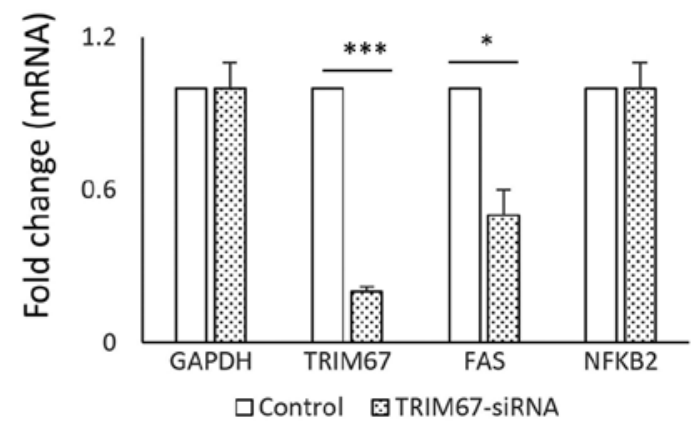

Figure 3. TRIM67 promotes NF-кB2 processing. (A) A549 cells were transfected with either control (empty vector) or TRIM67 plasmid. Proteins were harvested $24 \mathrm{~h}$ after transfection for western blot analysis. The results indicated that TRIM67 overexpression decreased the levels of p100, the precursor of

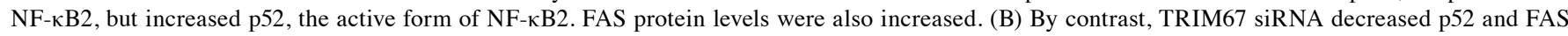
levels, but increased p100 protein levels. (C) A549 cells were transfected with either control (empty vector) or TRIM67 plasmid. Total RNA was extracted and qPCR was performed using GAPDH as the internal control. Student's t-test was used to evaluate the gene expression difference between two groups (control vs. TRIM67 plasmid). Results are expressed as the mean \pm standard deviation. ${ }^{*} \mathrm{P}<0.05$ and ${ }^{* * * *} \mathrm{P}<0.001$. (D) A549 cells were transfected with either control (non-targeting siRNA) or TRIM67 siRNA. Total RNA was extracted and qPCR performed to evaluate gene expression. GAPDH was used as internal control. Student's t-test was used to evaluate the gene expression difference between two groups (control vs. TRIM67 siRNA). Results are expressed as the mean \pm SD. ${ }^{*} \mathrm{P}<0.05$ and ${ }^{* * *} \mathrm{P}<0.001$. TRIM67, tripartite motif containing 67; NF- $\kappa \mathrm{B} 2, \mathrm{NF}-\kappa \mathrm{B}$ subunit 2; FAS, Fas cell surface death receptor; siRNA, small interfering RNA; qPCR, quantitative polymerase chain reaction.

were decreased, suggesting that GA-13315-induced apoptosis involved NF- $\kappa \mathrm{B}$-induced FAS (Fig. 4B and C). The effect of NBD peptide on TRIM67 and NFBK2 were also examined; the results indicated that neither mRNA nor protein levels were changed (Fig. 4B and C).

There is an $\mathrm{NF}-\kappa \mathrm{B}$ binding motif on the FAS promoter. A ChIP assay was used to investigate whether GA-13315 promoted $\mathrm{NF}-\kappa \mathrm{B}$ binding to the FAS promoter. As indicated in Fig. 4D, the binding of $\mathrm{NF}-\kappa \mathrm{B}$ to the FAS promoter was increased. A luciferase reporter assay was also used to determine whether GA-13315 activated FAS through NF- $\kappa$ B. Luciferase reporter plasmids containing the FAS promoter with or without $N F-\kappa B$ binding site were used. The results indicated that the FAS promoter activity was increased by 45 -fold in A549 cells and 25-fold in H460 cells upon GA-13315 treatment. Treatment with the NF- $\kappa \mathrm{B}$ inhibitor NDB peptide decreased the induction to 15 -fold. Deletion of NF- $\mathrm{NB}$ binding site also markedly decreased the luciferase activity (Fig. 4E), confirming the critical role of $\mathrm{NF}-\kappa \mathrm{B}$ in mediating GA-13315-induced FAS. Similar results were observed in the lung cancer H460 cells. Colelctively, the present study suggested that GA-13315 induced apoptosis via TRIM67, NF- $\kappa$ B2 and FAS. In addition, TRIM67 promoted the NF- $\kappa$ B pathway and enhanced FAS expression (Fig. 5).

\section{Discussion}

The present study identified 250 differentially expressed genes between GA-13315-treated A549 lung cancer cells and controls. TRIM67, NF-кB2 and FAS expression were confirmed to be increased at both the mRNA and protein levels upon GA-13315 treatment. Pathway analysis indicated a central role for the $\mathrm{NF}-\kappa \mathrm{B}$ complex in GA-13315-induced apoptosis. Additional investigation indicated that TRIM67 promoted NF- $\mathrm{B} 2$ gene processing, which in turn promoted the $\mathrm{NF}-\kappa \mathrm{B}$ pathway and subsequently increased FAS expression.

In order to gain a general understanding of the observed effects of GA-13315 in lung cancer, 2 distinct lung cancer cell lines (A549 and H460) were used. It was demonstrated that knockdown of TRIM67 resulted in a significant decrease of FAS and GA-13315-induced apoptosis, suggesting a critical role for TRIM67 in regulating apoptosis. In addition, the NF- $\kappa \mathrm{B}$ inhibitor (NBD peptide) also decreased GA-13315-induced apoptosis, suggesting a key role for $\mathrm{NF}-\kappa \mathrm{B}$ in GA-13315-induced apoptosis. Suppressing TRIM67 expression also inhibited the NF- $\kappa$ B pathway. TRIM67 knockdown decreased the active form of $\mathrm{NF}-\kappa \mathrm{B} 2$, p52, suggesting that TRIM67 promoted the processing of NF- $\kappa \mathrm{B} 2$ into its active form, p52, which may then enhance $N F-\kappa B$ signaling. As 
A

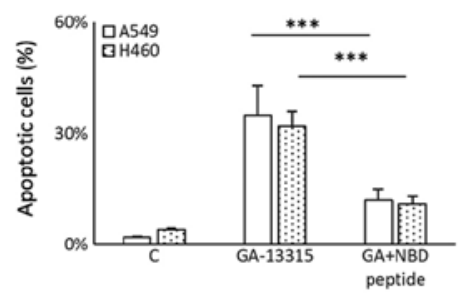

D
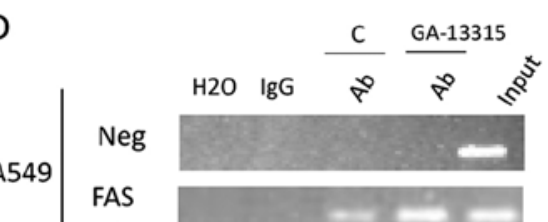
primer

$\mathrm{H} 460$

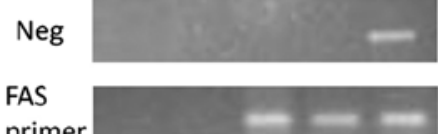

B

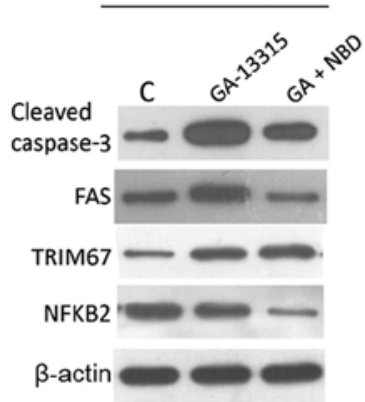

$\mathrm{H} 460$

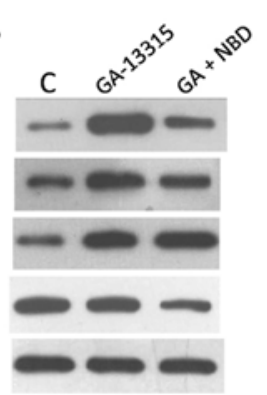

C
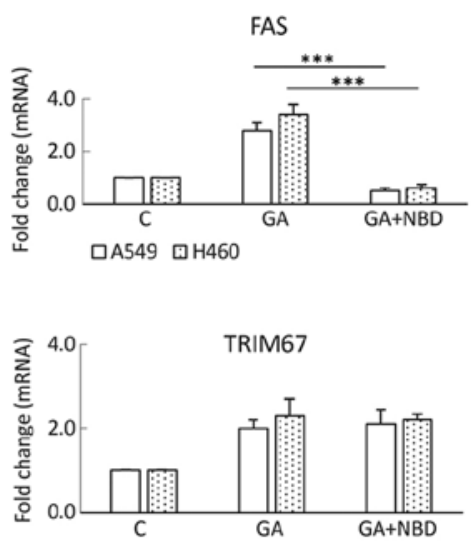

口A549 日H460

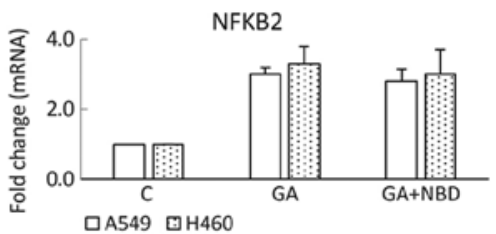

Figure 4. NF- $\kappa$ B mediates GA-13315-induced FAS and apoptosis. (A) NF- $\kappa$ B pathway inhibitor NBD peptide suppressed GA-13315-induced apoptosis, as measured by an Annexin V assay. The apoptosis rate was calculated by dividing Annexin V-positive cells by total cells. A one-way ANOVA followed by Tukey's honestly significant difference post-hoc test was used to evaluate the difference between the groups in each cell line. Results are expressed as the mean \pm SD. ${ }^{* * * *} \mathrm{P}<0.001$. (B) NBD peptide treatment decreased the levels of GA-13315-induced cleaved caspase-3. (C) A quantitative polymerase chain reaction assay indicated that NBD suppressed GA-13315-induced FAS mRNA expression, but exhibited no effects on the mRNA levels of TRIM67 and NF- $\kappa$ B2. (D) Chromatin immunoprecipitation demonstrated that GA-13315 treatment increased p52 binding to the FAS promoter. (E) A luciferase reporter vector containing the FAS promoter (FAS-luc) was used to evaluate activity changes in the FAS promoter. Cells treated with the control vehicle or GA-13315 were co-transfected with an empty vector or FAS-luc. Raw data were first normalized to the Renilla signal, and fold change was calculated by dividing the FAS-luc values by empty vector values. The results indicated that deletion of the NF- $\kappa$ B binding site on the FAS promoter decreased promoter activity induced by GA-13315. A one-way ANOVA followed by Tukey's honestly significant difference post-hoc test was used to evaluate the difference between groups in each cell line. Results are expressed as the mean \pm SD. ${ }^{*}<0.05$ and ${ }^{* * *} \mathrm{P}<0.001$. NBD, NEMO-binding domain; ANOVA, analysis of variance; SD, standard deviation; FAS, Fas cell surface death receptor; GA/GA-13315, 13-chlorine-3,15-dioxy-gibberellic acid methyl ester; TRIM67, tripartite motif containing 67; NF- $\mathrm{B} 2$, NF- $\mathrm{B}$ subunit 2; Ab, antibody; Neg, negative control.

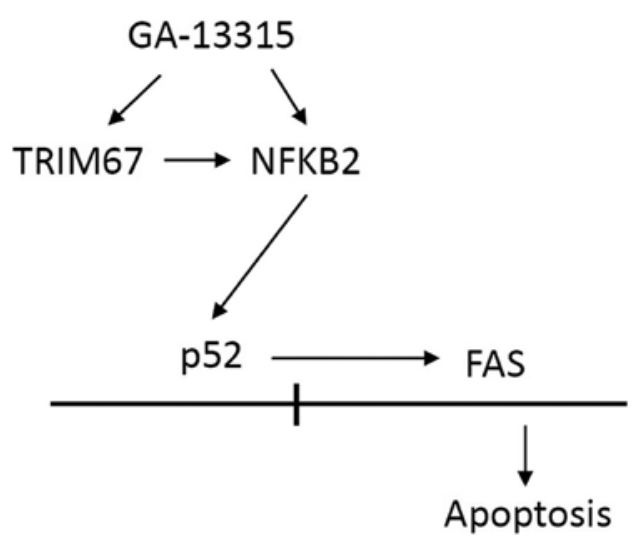

Figure 5. Proposed pathways mediating GA-13315-induced apoptosis.

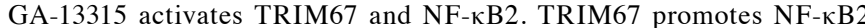
processing into the active form, NF- $\mathrm{kB} 2 \mathrm{p} 52$, which promotes FAS expression. GA-13315, 13-chlorine-3,15-dioxy-gibberellic acid methyl ester; TRIM67, tripartite motif containing 67; NF- $\mathrm{KB} 2, \mathrm{NF}-\kappa \mathrm{B}$ subunit 2; FAS, Fas cell surface death receptor.

GA-13315 already enhanced TRIM67 overexpression, the additional overexpression exhibited limited effects on p100 and p52 protein levels following exposure to GA13315 (data not shown). The role of NF-кB2 (p52) in mediating GA-13,315-induced FAS was demonstrated at multiple levels. Firstly, GA-13315 increased NF-kB2 (p52) binding to the FAS promoter. Secondly, deletion of the KB binding motif from the FAS promoter decreased GA-13315-induced FAS promoter activity in a luciferase reporter assay. Finally, inhibition of the NF- $\kappa B$ activator I $\kappa B$ kinase using NBD peptide decreased GA-13315-induced FAS expression. A number of chemopreventive compounds, in particular dietary compounds, including phenolic compounds, carotenoids, iridoids, nitrogen compounds, organosulfur compounds, phytosterols, essential oil compounds, polyunsaturated fatty acids and dietary fiber induce apoptosis of cancer cells. The proapoptotic properties of GA-13315 suggest potential chemopreventive effects.

TRIM67, located at chromosome 11q22-23, encodes a 783 amino acid protein with ListBox zinc-finger motifs and an adjacent leucine-zipper motif. TRIM67 was identified to be significantly downregulated in NSCLC cell lines (11), which was additionally confirmed by Hao et al (20), who suggested that TRIM67 may be a tumor suppressor gene. The functions of TRIM67 are largely unclear. Studies investigating TRIM protein members have indicated that TRIM proteins belong to a family of 
structurally conserved, rapidly evolving proteins $(21,22)$. TRIM proteins, as a type of E3 ubiquitin-linked enzyme, participate in cell cycle regulation, cell apoptosis, signal transduction, protein processing and transportation, proteasome-mediated protein degradation and responses to viral infection, all of which are important biological processes $(21,22)$. The results of the present study indicating that TRIM67 promoted the NF- $\kappa \mathrm{B}$ pathway are consistent with previous data that TRIM family members regulate the $\mathrm{NF}-\kappa \mathrm{B}$ pathway $(19,23)$.

The results of the present study are also consistent with the previous data that $\mathrm{NF}-\kappa \mathrm{B}$ regulates FAS transcription and FAS-mediated apoptosis (24). There are two types of $\mathrm{NF}-\kappa \mathrm{B}$ pathways, the canonical and non-canonical pathways, distinguished by the core components of $N F-\kappa B$ transcription factors (25). In the canonical pathway, following stimulation from Toll-like receptor ligands and inflammatory cytokines such as tumor necrosis factor and interleukin $1 \beta, \mathrm{NF}-\kappa \mathrm{B} 1$ (p50), which is processed from its precursor form $\mathrm{NF}-\kappa \mathrm{B} 1$ (p105), forms a heterodimer with transcription factor p65 and proto-oncogene c-Rel $(26,27)$. The non-canonical pathway is activated by ligands for lymphotoxin $\beta$ receptor, receptor activator of $\mathrm{NF}-\kappa \mathrm{B}$ and cluster of differentiation 40 , which induce $\mathrm{NF}-\kappa \mathrm{B} 2$ (p52) processing from the (p100) precursor and heterodimerization with transcription factor $\operatorname{RelB}(28,29)$. NF- $\kappa \mathrm{B}$ dimers recognize $\kappa \mathrm{B}$-binding motifs on the promoters of target genes, and then activate or repress their transcription in a context-dependent manner $(26,27)$. As NF- $\kappa \mathrm{B} 2$ belongs to the non-canonical $\mathrm{NF}-\kappa \mathrm{B}$ pathway, the results of the present study additionally suggested that the non-canonical NF- $\mathrm{B}$ pathway may similarly regulate apoptosis, and that TRIM67 is likely a new regulator of the non-canonical NF- $\kappa \mathrm{B}$ pathway, which warrants further investigation. $\mathrm{NF}-\kappa \mathrm{B}$ is involved in drug-induced apoptosis and the promotion of cancer aggressiveness, depending on the signaling context $(30,31)$, which may be the reason that the upregulation of $\mathrm{NF}-\kappa \mathrm{B}$ targeted genes, including tumor protein P53, was not observed in the RNA-Seq analysis of GA-13315-treated cells. Other TRIM family members may also interact with $\mathrm{NF}-\kappa \mathrm{B}$ to regulate apoptosis, which requires additional investigation.

The results of the present study also indicated that the apoptosis rate of A549 cells increased significantly along with the increase in FAS expression levels following treatment with GA-13315. However, various death receptors have been identified to serve a role in apoptosis signaling. Tumor necrosis factor receptor 1 (32), cytopathic avian receptor 1 (33), nerve growth factor receptor (34), death receptor (DR)3 (35), DR4 and DR5 (36) are present, in addition to the most common FAS/FASL. Whether other cell death pathways are also involved requires further investigation. Similarly, TRIM67 may regulate other signaling pathways to modulate GA-13315-induced apoptosis. TRIM67 has been demonstrated to modulate the RAS signaling pathway in neural cells (37). Whether the TRIM67-RAS signaling pathway is involved in regulating GA-13315-induced apoptosis also requires further investigation.

The present study revealed novel mechanisms underlying GA-13315-induced apoptosis. Due to limited resources, the experimental design may not be ideally rigorous, and therefore, the results should be interpreted with caution. For example, there might be other mechanisms for GA-13315-induced apoptosis that require verification. The evaluation of
GA-13315-induced apoptosis in additional cancer cell lines may provide an improved understanding of the mechanisms involved. How GA-13315 increases TRIM67 and NF- $\kappa$ B2 gene expression, and how TRIM67 regulates NF- $\kappa \mathrm{B} 2$ processing, require additional detailed investigation.

The present study indicated that GA-13315-induced apoptosis involved TRIM67, NF-кB2 and FAS. TRIM67 promoted the NF- $\kappa \mathrm{B}$ pathway and enhanced FAS expression, which may be an underlying mechanism for GA-13315-induced apoptosis in lung cancer cells.

\section{Acknowledgements}

Not applicable.

\section{Funding}

The present study was supported by the National Natural Science Foundation of China (grant no. 81460559) and the Key Scientific and Technological Achievements Cultivation Project of Kunming Medical University (grant no. CGPY201502).

\section{Availability of data and materials}

The RNA-Seq data, and qPCR primer sequence and pathway analysis results are included as supplemental data (Tables SI and SII, Figs. S1, S2 and S3). All other data and materials are available upon request.

\section{Authors' contributions}

JC synthesized and provided the compound GA-13315. CQ, JC and RL conceived and supervised the study and RL and CQ designed the experiments. CQ, RL and YC performed the experiments, and $\mathrm{YC}$ provided reagents. TS and $\mathrm{JH}$ analyzed the data. $\mathrm{RL}, \mathrm{YC}$ and JH wrote the manuscript, and CQ, JC and YC edited and revised the manuscript. All authors discussed and reviewed the results, and approved the final version of the manuscript.

\section{Ethics approval and consent to participate}

Not applicable.

\section{Patient consent for publication}

Not applicable.

\section{Competing interests}

The authors declare that they have no competing interests.

\section{References}

1. Negoita S, Feuer EJ, Mariotto A, Cronin KA, Petkov VI, Hussey SK, Benard V, Henley SJ, Anderson RN, Fedewa S, et al: Annual report to the nation on the status of cancer, part II: Recent changes in prostate cancer trends and disease characteristics. Cancer 124: 2801-2814, 2018.

2. Cronin KA, Lake AJ, Scott S, Sherman RL, Noone AM, Howlader N, Henley SJ, Anderson RN, Firth AU, Ma J, et al: Annual report to the nation on the status of cancer, part I: National cancer statistics. Cancer 124: 2785-2800, 2018. 
3. Lam VK and Papadimitrakopoulou V: Master protocols in lung cancer: Experience from lung master protocol. Curr Opin Oncol 30: 92-97, 2018.

4. Lemjabbar-Alaoui H, Hassan OU, Yang YW and Buchanan P: Lung cancer: Biology and treatment options. Biochim Biophys Acta 1856: 189-210,2015.

5. Chen J, Sun Z, Zhang Y, Zeng X, Qing C, Liu J, Li L and Zhang H: Synthesis of gibberellin derivatives with anti-tumor bioactivities. Bioorg Med Chem Lett 19: 5496-5499, 2009.

6. Zhang Y, Zhang H, Chen J, Zhao H, Zeng X, Zhang $\mathrm{H}$ and Qing C: Antitumor and antiangiogenic effects of GA-13315, a gibberellin derivative. Invest New Drugs 30: 8-16, 2012.

7. Mo J, Kang M, Ye JX, Chen JB, Zhang HB and Qing C: Gibberellin derivative GA-13315 sensitizes multidrug-resistant cancer cells by antagonizing ABCB1 while agonizes ABCC1. Cancer Chemother Pharmacol 78: 51-61, 2016.

8. Shen S and Tang J: Effects and mechanism of GA-13315 on the proliferation and apoptosis of KB cells in oral cancer. Oncol Lett 14: 1460-1463, 2017.

9. Mayer RE, Hendrix P, Cron P, Matthies R, Stone SR, Goris J, Merlevede W, Hofsteenge J and Hemmings BA: Structure of the $55-\mathrm{kDa}$ regulatory subunit of protein phosphatase 2A: Evidence for a neuronal-specific isoform. Biochemistry 30: 3589-3597, 1991.

10. Majchrzak-Celińska A, Słocińska M, Barciszewska AM, Nowak S and Baer-Dubowska W: Wnt pathway antagonists, SFRP1, SFRP2, SOX17, and PPP2R2B, are methylated in gliomas and SFRP1 methylation predicts shorter survival. J Appl Genet 57: 189-197, 2016.

11. Zhan W, Han T, Zhang C, Xie C, Gan M, Deng K, Fu M and Wang JB: TRIM59 promotes the proliferation and migration of non-small cell lung cancer cells by upregulating cell cycle related proteins. PLoS One 10: e0142596, 2015.

12. Dimitrakopoulos FD, Antonacopoulou AG, Kottorou AE, Maroussi S, Panagopoulos N, Koukourikou I, Scopa C, Kalofonou M, Koutras A, Makatsoris T, et al: NF- $\kappa$ B2 genetic variations are significantly associated with non-small cell lung cancer risk and overall survival. Sci Rep 8: 5259, 2018.

13. Siena L, Pace E, Ferraro M, Di Sano C, Melis M, Profita M, Spatafora $M$ and Gjomarkaj $M$ : Gemcitabine sensitizes lung cancer cells to Fas/FasL system-mediated killing. Immunology 141: 242-255, 2014.

14. Mou H, Moore J, Malonia SK, Li Y, Ozata DM, Hough S, Song CQ, Smith JL, Fischer A, Weng Z, et al: Genetic disruption of oncogenic Kras sensitizes lung cancer cells to fas receptor-mediated apoptosis. Proc Natl Acad Sci USA 114: 3648-3653, 2017.

15. Kim D, Pertea G, Trapnell C, Pimentel H, Kelley R and Salzberg SL: TopHat2: Accurate alignment of transcriptomes in the presence of insertions, deletions and gene fusions. Genome Biol 14: R36, 2013.

16. Seyednasrollah F, Laiho A and Elo LL: Comparison of software packages for detecting differential expression in RNA-seq studies. Brief Bioinform 16: 59-70, 2015.

17. Livak KJ and Schmittgen TD: Analysis of relative gene expression data using real-time quantitative PCR and the 2(-Delta Delta C(T)) method. Methods 25: 402-408, 2001.

18. Savinova OV, Hoffmann A and Ghosh G: The Nfkb1 and $\mathrm{Nfkb} 2$ proteins p105 and p100 function as the core of high-molecular-weight heterogeneous complexes. Mol Cell 34: 591-602, 2009

19. Rajsbaum R, García-Sastre A and Versteeg GA: TRIMmunity: The roles of the TRIM E3-ubiquitin ligase family in innate antiviral immunity. J Mol Biol 426: 1265-1284, 2014.

20. Hao L, Du B and Xi X: TRIM59 is a novel potential prognostic biomarker in patients with non-small cell lung cancer: A research based on bioinformatics analysis. Oncol Lett 14 2153-2164, 2017.
21. Meroni G and Diez-Roux G: TRIM/RBCC, a novel class of 'single protein RING finger' E3 ubiquitin ligases. Bioessays 27: 1147-1157, 2005.

22. Esposito D, Koliopoulos MG and Rittinger K: Structural determinants of TRIM protein function. Biochem Soc Trans 45 183-191, 2017.

23. Uchil PD, Hinz A, Siegel S, Coenen-Stass A, Pertel T, Luban J and Mothes W: TRIM protein-mediated regulation of inflammatory and innate immune signaling and its association with antiretroviral activity. J Virol 87: 257-272, 2013.

24. Liu F, Bardhan K, Yang D, Thangaraju M, Ganapathy V, Waller JL, Liles GB, Lee JR and Liu K: NF- $\kappa$ B directly regulates fas transcription to modulate fas-mediated apoptosis and tumor suppression. J Biol Chem 287: 25530-25540, 2012.

25. Pomerantz JL and Baltimore D: Two pathways to NF-kappaB. Mol Cell 10: 693-695, 2002.

26. Gilmore TD: Introduction to NF-kappaB: Players, pathways, perspectives. Oncogene 25: 6680-6684,2006.

27. Hayden MS and Ghosh S: Shared principles in NF-kappaB signaling. Cell 132: 344-362, 2008

28. Cildir G, Low KC and Tergaonkar V: Noncanonical NF- $\kappa B$ signaling in health and disease. Trends Mol Med 22: 414-429, 2016

29. Sun SC: The noncanonical NF- $\mathrm{BB}$ pathway. Immunol Rev 246 : 125-140, 2012.

30. Müller I, Beissert S and Kulms D: Anti-apoptotic NF-kB and 'gain of function' mutp53 in concert act pro-apoptotic in response to UVB+IL-1 via enhanced TNF production. J Invest Dermatol 135: 851-860, 2015.

31. Kaltschmidt B, Kaltschmidt C, Hofmann TG, Hehner SP, Dröge W and Schmitz ML: The pro-or anti-apoptotic function of NF-kappaB is determined by the nature of the apoptotic stimulus. Eur J Biochem 267: 3828-3835, 2000.

32. You BR, Han BR and Park WH: Suberoylanilide hydroxamic acid increases anti-cancer effect of tumor necrosis factor- $\alpha$ through up-regulation of TNF receptor 1 in lung cancer cells. Oncotarget 8: 17726-17737, 2017.

33. Brojatsch J, Naughton J, Rolls MM, Zingler K and Young JA: CAR1, a TNFR-related protein, is a cellular receptor for cytopathic avian leukosis-sarcoma viruses and mediates apoptosis. Cell 87: 845-855, 1996.

34. Zhou X, Hao Q, Liao P, Luo S, Zhang M, Hu G, Liu H, Zhang Y, Cao B, Baddoo M, et al: Nerve growth factor receptor negates the tumor suppressor p53 as a feedback regulator. Elife 5: pii: e15099, 2016.

35. Choi KE, Hwang CJ, Gu SM, Park MH, Kim JH, Park JH, Ahn YJ, Kim JY, Song MJ, Song HS, et al: Cancer cell growth inhibitory effect of bee venom via increase of death receptor 3 expression and inactivation of NF-kappa B in NSCLC cells. Toxins (Basel) 6: 2210-2228, 2014

36. Chen M, Wang X, Zha D, Cai F, Zhang W, He Y, Huang Q, Zhuang $\mathrm{H}$ and Hua ZC: Apigenin potentiates TRAIL therapy of non-small cell lung cancer via upregulating DR4/DR5 expression in a p53-dependent manner. Sci Rep 6: 35468, 2016.

37. Yaguchi H, Okumura F, Takahashi H, Kano T, Kameda $\mathrm{H}$, Uchigashima M, Tanaka S, Watanabe M, Sasaki H and Hatakeyama S: TRIM67 protein negatively regulates ras activity through degradation of $80 \mathrm{~K}-\mathrm{H}$ and induces neuritogenesis. J Biol Chem 287: 12050-12059, 2012. 